\title{
Investigating the relationship between UMODL1 gene polymorphisms and high myopia: a case-control study in Chinese
}

\author{
Miao-miao Zhu ${ }^{1,2,3}$, Maurice KH Yap ${ }^{3}$, Daniel WH Ho ${ }^{2,3}$, Wai Yan Fung ${ }^{2}$, Po Wah Ng ${ }^{2,3}$, Yang-shun Gu' \\ and Shea Ping Yip ${ }^{2^{*}}$
}

\begin{abstract}
Background: The UMODL1 gene was found to be associated with high myopia in Japanese. This study aimed to investigate this gene for association with high myopia in Chinese.

Methods: Two groups of unrelated Han Chinese from Hong Kong were recruited using the same criteria: Sample Set 1 comprising 356 controls (spherical equivalent, SE, within \pm 1 diopter or D) and 356 cases (SE $\leq-8 D$ ), and Sample Set 2 comprising 394 controls and 526 cases. Fifty-nine tag single nucleotide polymorphisms (SNPs) were selected and genotyped for Sample Set 1. Four SNPs were followed up with Sample Set 2. Both single-marker and haplotype analyses were performed with cases defined by different SE thresholds. Secondary phenotypes were also analyzed for association with genotypes.

Results: Data filtering left 57 SNPs for analysis. Single-marker analysis did not reveal any significant differences between cases and controls in the initial study. However, haplotype GCT for markers rs220168-rs220170-rs11911271 showed marginal significance (empirical $P=0.076$; SE $\leq-12 \mathrm{D}$ for cases), but could not be replicated in the follow-up study. In contrast, non-synonymous SNP rs3819142 was associated with high myopia (SE $\leq-10 D)$ in the follow-up study, but could not be confirmed using Sample Set 1. The SNP rs2839471, positive in the original Japanese study, gave negative results in all our analyses. Exploratory analysis of secondary phenotypes indicated that allele $C$ of rs220120 was associated with anterior chamber depth (adjusted $P=0.0460$ ).
\end{abstract}

Conclusions: Common UMODL1 polymorphisms were unlikely to be important in the genetic susceptibility to high myopia in Han Chinese.

Keywords: High myopia, UMODL1, Single nucleotide polymorphism, Association study, Secondary phenotype

\section{Background}

Myopia is the commonest eye defect worldwide. Myopic eyes focus the image of a distant object in front of the retina when accommodation is relaxed. High myopia is often defined as a refractive error of -6.0 diopters (D) or worse, and severely myopic eyes are particularly vulnerable to many ocular pathologies [1]. The increasing prevalence and decreasing earlier age-of-onset of myopia make it a priority in many populations [2]. The prevalence

\footnotetext{
*Correspondence: shea.ping.yip@inet.polyu.edu.hk

${ }^{2}$ Department of Health Technology and Informatics, The Hong Kong

Polytechnic University, Hong Kong SAR, China

Full list of author information is available at the end of the article
}

of myopia is much higher in Asian populations ( 70\%) than in Caucasian populations (15\%-27\%) [2,3].

Myopia is a complex disease caused by both genetic and environmental factors plus their interactions although the exact etiology is still unclear [4,5]. High heritability observed in many twin and family studies provides convincing evidence that genetic factors play a strong role in the development of myopia [6,7]. Genetic association studies are more powerful than linkage analysis in detecting genes with relatively small effects in complex diseases [5]. While genome-wide association studies are getting more popular, the cost is still prohibitive for many researchers. Candidate gene studies remain important for hunting predisposing factors for complex diseases.

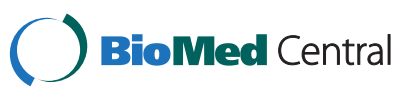


Recently, a Japanese group performed a genome-wide case-control association analysis of high myopia with 27,000 microsatellite markers and followed up a positive marker (D21S0083i on chromosome 21q22.3) with 39 single nucleotide polymorphisms (SNPs) in a casecontrol study [8]. The SNP rs2839471 was found to be associated with high myopia even after correction for multiple comparisons by Bonferroni procedure $(P=0.00027$ and corrected $P=0.01$ ). The uromodulin-like 1 (UMODL1) gene harboring rs2839471 was suggested as a new susceptibility gene for high myopia.

The UMODL1 gene (GeneID: 89766) is a proteincoding gene located at chromosome 21q22.3, spans a genomic region of $\sim 80 \mathrm{~kb}$ and consists of 23 exons (http://www.ncbi.nlm.nih.gov/). It encodes several transcripts via alternative splicing, among which UMODL1S and $U M O D L 1 L$ are the two major isoforms that have been better characterized so far. Both isoforms contain multiple domains such as whey acidic protein, calciumbinding EGF-like, fibronectin type III, SEA (sea urchin sperm protein, enterokinase, and agrin), and zona pellucida domains [9]. These domains are typically found in various combinations in extracellular matrix (ECM) proteins, indicating that the UMODL1 protein may be secreted and associated with ECM proteins involved in cell/cell and cell/ECM adhesion and in cell migration [10]. The gene is expressed at low level in the eye [11].

The present study aimed to systematically investigate the UMODL1 gene for association with high myopia in a Han Chinese population. We performed with a Sample Set 1 an initial case-control study to identify putatively positive SNPs or haplotypes and then replicated the "positive" results by an independent Sample Set 2.

\section{Methods}

\section{Subjects and DNA samples}

Two case-control sample sets were used in this study and collected using the same entry criteria as described previously [12]. Each study subject received a complete ocular examination. Spherical equivalent $(\mathrm{SE})$ within $\pm 1.0 \mathrm{D}$ categorized a subject as an emmetropic control while SE of $-8.00 \mathrm{D}$ or less defined a subject as a case with high myopia. The study was approved by the Human Subjects Ethics Subcommittee of the Hong Kong Polytechnic University, and adhered to the tenets of the Declaration of Helsinki. Written informed consents were obtained from all subjects. DNA was extracted from whole blood with a modified salt precipitation method [12] or FlexiGene DNA Kit (Qiagen, Hilden, Germany) according to the manufacturer's instructions.

\section{Selection of tag SNPs}

In the initial study, 58 tag SNPs were selected from an $86-\mathrm{kb}$ genomic region comprising the UMODL1 gene and its 3-kb flanking regions (both upstream and downstream) by Haploview [13]. The selection was based on the Han Chinese data from the International HapMap Project (release 23a, phase II; http://www.hapmap.org/) [14] with the following criteria: pairwise tagging algorithm, $\mathrm{r}^{2} \geq 0.8$, minor allele frequency (MAF) $>0.2$ and forced inclusion of SNPs that had been tested in the Japanese study. A non-synonymous SNP (rs3819142) was added at a later stage and genotyped for both sample sets because it was in moderate linkage disequilibrium (LD; $r^{2}=0.6$ ) with $r 220170$, which was one of the SNPs constituting a putatively positive haplotype from the initial study.

\section{Genotyping of SNPs}

Three different methods were used to genotype these SNPs: mass spectrometry of multiplexed primer-extended products [15] (MassArray iPLEX; Sequenom, San Diego, $\mathrm{CA})$, restriction fragment length polymorphism (RFLP), and denaturing high performance liquid chromatography (DHPLC) of primer-extended products [16].

In the initial study, 56 SNPs were grouped together with SNPs of other on-going studies and genotyped by a mass spectrometric method according to the manufacturer's instructions (Sequenom), as reported previously by our group [17-19]. Sequences of primers for PCR and primer extension (PE) are shown in Additional file 1. Two SNPs (rs220140 and rs220173) were genotyped by RFLP because they could not be grouped together with other SNPs for genotyping by the iPLEX assays. Polymerase chain reaction (PCR) was performed in a $10-\mu \mathrm{l}$ reaction mixture containing $10 \mathrm{ng}$ of DNA template, 0.3 $\mu \mathrm{M}$ or $0.5 \mu \mathrm{M}$ of each primer (Additional file 2), 0.2 $\mathrm{mM}$ of each dNTP and $0.2 \mathrm{U}$ of HotStarTaq Plus DNA Polymerase (Qiagen, Hilden, Germany) in $1 \times$ PCR buffer (with $1.5 \mathrm{mM} \mathrm{MgCl}_{2}$, Qiagen). Amplification was performed in a 96-well PCR machine (GeneAmp PCR system 9700; Applied Biosystems, Foster City, CA). The PCR program was as follows: initial denaturation at $95^{\circ} \mathrm{C}$ for $5 \mathrm{~min}$, followed by 35 cycles of denaturation at $95^{\circ} \mathrm{C}$ for 30 seconds, annealing at $60^{\circ} \mathrm{C}$ for 30 seconds and elongation at $72^{\circ} \mathrm{C}$ for 30 seconds, plus a final extension at $72^{\circ} \mathrm{C}$ for 5 minutes. PCR products were digested overnight with a restriction enzyme (MBI Fermentas, Vilnius, Lithuania; Additional file 2) according to the manufacturer's instructions. Digested products were pre-stained for 15 minutes with SYBR Green I (Invitrogen, Carlsbad, CA) and then separated in 8\% 102-well horizontal polyacrylamide gels prepared using a home-made casting cassette.

In the follow-up study, four SNPs were genotyped. Of these, rs220168, rs220170 and rs3819142 were first genotyped by the mass spectrometric method (MassArray iPLEX). Samples that could not be grouped together and 
genotyped within a SpectroCHIP (for 360 samples; Sequenom) were genotyped by RFLP. Either way, the methods were as described above (Additional file 1 and Additional file 2). On the other hand, rs11911271 was genotyped by PE coupled with DHPLC [16]. The PCR templates were generated using the same conditions as mentioned above and purified by shrimp alkaline phosphatase and exonuclease I (New England Biolabs, Beverly, MA). PE reactions were performed in a $20-\mu$ l reaction mixture containing $10 \mu \mathrm{l}$ of purified PCR products, 1.5 $\mu \mathrm{M}$ of extension primer (Additional file 2), $50 \mu \mathrm{M}$ of ddCTP/ddTTP and 1 unit of Therminator (New England Biolabs, Beverly, MA) in a $1 \times$ reaction buffer supplied by the manufacturer. Thermocycling was performed with an initial denaturation at $96^{\circ} \mathrm{C}$ for 1 minute, followed by 55 cycles of $96^{\circ} \mathrm{C}$ for 10 seconds, $43^{\circ} \mathrm{C}$ for 15 seconds and $60^{\circ} \mathrm{C}$ for 1 minute. PE products were analyzed by DHPLC (WAVE Nucleic Acid Fragment Analysis System; Transgenomic, Omaha, NE) as described previously [20] with $20 \%$ starting buffer B concentration.

\section{Statistical analysis}

Ocular data were analyzed using the SPSS package (version 11.5; SPSS Inc.). Subjects were classified as affected (cases) or unaffected (controls). High myopia was analyzed as a dichotomous trait and a quantitative trait separately. Subset analysis was also performed by defining cases with increasingly restrictive thresholds of refractive error in order to fully explore the genotype data (Sample Sets 1 and 2). Hardy-Weinberg equilibrium (HWE) was tested for controls and cases separately by Fisher's exact test executed within Plink (version 1.07) [21]. SNPs that were not in HWE were excluded from subsequent association analysis. Haplotype blocks were constructed by Haploview with an algorithm known as the solid spine of linkage disequilibrium (SSLD) [13]. Single-marker and haplotype analyses were performed with three packages: Haploview, Plink and Beagle (ver. 3.0) [22-24]. Multiple testing was corrected by 10,000 permutations to generate empirical $P$ values $\left(P_{\text {emp }}\right)$. Localized haplotype clusters that were found significantly associated with high myopia by Beagle were further analyzed using the cluster2haps program to identify the haplotypes and SNPs defining the clusters [24].

The SPREG program [25] was used to analyze secondary phenotypes because the case-control subjects were not collected from a random population. SPREG implements valid and efficient statistical methods for analyzing secondary phenotypes collected in case-control association studies. It used a modified linear regression method to analyze secondary phenotypes including axial length (AXL), anterior chamber depth (ACD), lens thickness (LT) and corneal power $(\mathrm{CP})$. False discovery rate (FDR) was used to correct for multiple comparisons within a given secondary phenotype [26].

\section{Results}

\section{Analysis of ocular data}

The initial study recruited 712 unrelated Southern Han Chinese subjects (Sample Set 1) including 356 controls and 356 cases defined by SE $\leq-8.0 \mathrm{D}$ (Table 1). SNPs constituting positive haplotypes from the initial study were replicated using a second sample set (Sample Set 2). Sample Set 2 consisted of 920 unrelated Han Chinese subjects with 394 controls and 526 cases defined by SE $\leq-8.0 \mathrm{D}$ (Table 1). There were fewer females in the control group than in the case group: $56.2 \%$ versus $69.7 \%$ for Sample Set $1(P=0.0027)$, and $59.1 \%$ versus $67.8 \%$ for Sample Set $2(P=0.0093)$. The control subjects were on average younger than the case subjects in Sample Set 1 (26.0 versus 28.9 years, $\mathrm{P}<0.0001$ ) while both subject groups were of similar age in Sample Set 2 (32.9 versus 32.6 years, $P=0.5632$ ). For both sample sets, the controls and the cases had very similar values for anterior chamber depth, lens thickness and corneal power.

Table 1 Characteristics of study subjects for sample Set 1 and Set $2^{*}$

\begin{tabular}{|c|c|c|c|c|c|c|c|c|}
\hline \multirow[b]{3}{*}{ Characteristic } & \multicolumn{4}{|c|}{ Sample Set 1} & \multicolumn{4}{|c|}{ Sample Set 2} \\
\hline & \multicolumn{2}{|l|}{ Controls } & \multicolumn{2}{|l|}{ Cases } & \multicolumn{2}{|l|}{ Controls } & \multicolumn{2}{|l|}{ Cases } \\
\hline & $\pm 1 D$ & $\leq-8 D$ & $\leq-10 D$ & $\leq-12 \mathrm{D}$ & $\pm 1 D$ & $\leq-8 D$ & $\leq-10 D$ & $\leq-12 \mathrm{D}$ \\
\hline Total no. & 356 & 356 & 206 & 93 & 394 & 526 & 269 & 116 \\
\hline Females, no. (\%) & $200(56.2)$ & $248(69.7)$ & $144(69.9)$ & $67(72.0)$ & $233(59.1)$ & $356(67.8)$ & $194(72.1)$ & $84(72.4)$ \\
\hline Age, mean (SD), y & $26.0(7.1)$ & $28.9(7.8)$ & $29.6(7.8)$ & $31.3(8.3)$ & $32.9(9.8)$ & $32.6(8.9)$ & $32.6(8.6)$ & $32.6(9.0)$ \\
\hline Spherical equivalent, mean (SD), D & $0.01(0.46)$ & $-10.65(2.64)$ & $-11.98(2.73)$ & $-13.76(3.15)$ & $0.13(0.54)$ & $-10.29(2.31)$ & $-11.59(2.37)$ & $-13.50(2.34)$ \\
\hline Axial length, mean (SD), mm & $23.83(0.82)$ & $27.81(1.17)$ & $28.19(1.17)$ & $28.70(1.23)$ & $23.69(0.82)$ & $27.60(1.21)$ & $28.04(1.24)$ & $28.68(1.34)$ \\
\hline Anterior chamber depth, mean (SD), mm & $3.57(0.34)$ & $3.67(0.35)$ & $3.66(0.36)$ & $3.58(0.37)$ & $3.20(0.41)$ & $3.37(0.39)$ & $3.33(0.40)$ & $3.33(0.43)$ \\
\hline Lens thickness, mean (SD), mm & $3.98(0.48)$ & $4.06(0.55)$ & $4.08(0.56)$ & $4.10(0.50)$ & $4.30(0.56)$ & $4.22(0.53)$ & $4.24(0.54)$ & $4.26(0.61)$ \\
\hline Corneal power, mean (SD), D & $43.54(1.50)$ & $44.50(1.40)$ & $44.64(1.38)$ & $44.87(1.40)$ & $44.04(1.49)$ & $44.76(1.47)$ & $44.83(1.49)$ & $44.99(1.45)$ \\
\hline
\end{tabular}

* The table shows the data for the right eyes only. 
Table 1 also shows the summary of cases defined by different thresholds of SE. For Sample Set 1, the number of cases reduced from 356 to 206 to 93 when the threshold SE changed from $-8 \mathrm{D}$ to $-10 \mathrm{D}$ to $-12 \mathrm{D}$. The mean SE became more negative (from $-10.65 \mathrm{D}$ to $-11.98 \mathrm{D}$ to $-13.76 \mathrm{D}$ ), and the axial length longer (from $27.81 \mathrm{~mm}$ to $28.19 \mathrm{~mm}$ to $28.70 \mathrm{~mm}$ ) with increasingly stringent thresholds for cases. Similar trends of changes were observed for Sample Set 2 (Table 1).

\section{Initial association study}

The 58 tag SNPs selected on the basis of the Han Chinese data from the HapMap database could capture the genetic information for a total of 125 SNPs in the indicated region $(86.0 \mathrm{~kb})$ at a mean $\mathrm{r}^{2}$ of 0.971 . The nonsynonymous SNP rs3819142, which involves an amino acid change (asparagine to histidine), was added as has been explained above. Of the 59 SNPs genotyped, one SNP (rs1571737) was removed because it failed to pass the minimum $80 \%$ call rate for the MassARRAY iPLEX assay. Another SNP (rs220279) was also removed because the genotypes were not in HWE in the control group $(P<0.001)$. All the remaining 57 SNPs were in HWE at a threshold $P$ value of 0.001 in both controls and cases. These 57 SNPs were designated as S1 to S57 in a sequential order from the 5' end to the 3 ' end of the sense strand of the UMODL1 gene for the sake of easy referencing (Table 2). Based on the SSLD algorithm of Haploview, 16 haplotype blocks were constructed (Additional file 3). The overall LD among the 57 SNPs under analysis was quite weak.

Table 2 summarizes the distribution of genotypes, minor allele frequencies and the results of single-marker association analysis performed with Plink. In particular, no association between high myopia and any SNP was detected under any of the five genetic models tested (allelic, genotypic, additive, dominant and recessive) after correcting for multiple comparisons. The conclusion remained the same even if sex and age were added as covariates for adjustment to avoid their potential confounding effects. Even when the threshold SE for defining cases was more restrictive at $-10 \mathrm{D}$ or $-12 \mathrm{D}$, none of the 57 SNPs gave a significant difference between cases and controls after 10,000 permutations. The results were consistent with those generated by the Beagle package (data not shown). The SNP (rs2839471 or S46) that was positive in the Japanese study [8] was found negative in all our analyses.

Haplotype analysis still did not show any significant differences between cases and controls when it was conducted using either LD-block-based model by Haploview or a sliding window strategy by Plink (data not shown in either setting). The same conclusion was drawn when sex and age were added as covariates in the analysis by
Plink. However, one haplotype cluster defined by the 0.G allele of rs220168 (S47) showed a marginally significant result $\left(P_{\mathrm{emp}}=0.0763\right.$ only for cases with $\left.\mathrm{SE} \leq-12 \mathrm{D}\right)$ when tested with the Beagle program by a localized haplotype-cluster model and corrected for multiple testing by 10,000 permutations. For rs220168 (S47), the designation "0.G" represented the $G$ allele of the marker (rs220168) at node "0" (zero) in a direct acyclic graph used by Beagle to represent localized haplotype clusters $[22,23]$. The SNP rs220168 (S47) was in the same LD block as the Japanese positive SNP rs2839471 (S46) (Additional file 3), and the two SNPs are 115 bp apart. Therefore, we further identified the haplotypes that were present in this cluster with the cluster2haps program. The haplotype GCT (211 in the 1-2 or major-minor format) for markers rs220168-rs220170-rs11911271 (S47S48-S49) showed the most significant association: $P=0.0015$ with an odds ratio (OR) of 3.78 when compared with all other haplotypes (Table 3). According to the HapMap data, the non-synonymous SNP rs3819142 (S54) was in moderate LD $\left(r^{2}=0.6\right)$ with $\mathrm{rs} 220170$ (S48). It might have some functional consequences leading to high myopia. Therefore, these SNPs were further tested in a follow-up study using Sample Set 2: rs220168 (S47), rs220170 (S48), rs11911271 (S49) and rs3819142 (S54).

\section{Follow-up association study}

The genotypes of the four SNPs that were followed up using Sample Set 2 were all in HWE. The SNPs rs220168 (S47, asymptotic $P$ value $\left.\left(P_{\text {asym }}\right)=0.0074\right)$ and rs3819142 (S54, $\left.P_{\text {asym }}=0.0021\right)$ were associated with extreme myopia defined by $\mathrm{SE} \leq-10 \mathrm{D}$ under a dominant genetic model (Table 4) while there was no significant finding with cases defined by $\mathrm{SE} \leq-8 \mathrm{D}$ or $\leq-12 \mathrm{D}$. Only the result of rs3819142 (S54) remained significant $\left(P_{\mathrm{emp}}=0.0168\right)$ after correction for multiple comparisons (Table 4). The conclusion remained valid when sex and age were included as covariates because the $P$ values only fluctuated slightly. Since these four SNPs did not show any significant association with high myopia at any of the SE thresholds tested in Sample Set 1 (see above and Table 2), we performed a combined analysis of these two sample sets for these four SNPs with cases defined by $\mathrm{SE} \leq-10 \mathrm{D}$. The association signal for rs3819142 (S54) disappeared in the combined analysis (Table 4). Haplotype analysis did not reveal any significant finding either (data not shown). In other words, the overall results indicated that rs3819142 (S54) was not associated with high myopia.

Interestingly, analysis of Sample Set 2 by Beagle also showed that the haplotype cluster 0.C allele of rs3819142 (S54) was associated with extreme myopia defined by SE $\leq$ $-10 \mathrm{D}$ (Table 3). Upon exploring the cluster with the cluster2haps program, it was identified that the single marker 
Table 2 Summary statistics of UMODL1 SNPs in initial study (cases defined as spherical equivalent $\leq \mathbf{- 8 . 0}$ diopters)

\begin{tabular}{|c|c|c|c|c|c|c|c|c|c|c|c|}
\hline \multirow{2}{*}{ SNP* } & & \multirow[b]{2}{*}{ Location* } & \multicolumn{2}{|c|}{ Allelet } & \multicolumn{2}{|c|}{ Genotype counts $(11 / 12 / 22)$} & \multicolumn{2}{|c|}{ Minor allele freq } & \multicolumn{3}{|c|}{ Association test (best result) $\ddagger$} \\
\hline & & & 1 & 2 & Controls & Cases & Controls & Cases & $P_{\text {asym }}$ & Model & $P_{\text {emp }}$ \\
\hline rs 220260 & S1 & Intergenic & C & G & $149 / 157 / 44$ & $155 / 160 / 36$ & 0.3500 & 0.3343 & 0.3630 & Recessive & 1 \\
\hline rs220262 & $\mathrm{S} 2$ & Intergenic & A & $\mathrm{T}$ & $174 / 148 / 29$ & $173 / 157 / 22$ & 0.2934 & 0.2855 & 0.3038 & Recessive & 1 \\
\hline rs 220263 & S3 & Intergenic & A & G & $106 / 159 / 83$ & $85 / 179 / 86$ & 0.4670 & 0.4986 & 0.0673 & Dominant & 0.9882 \\
\hline rs 220265 & S4 & Intergenic & C & A & $127 / 155 / 62$ & $106 / 181 / 65$ & 0.4055 & 0.4418 & 0.0572 & Dominant & 0.9763 \\
\hline rs12627387 & S5 & Intergenic & G & A & $100 / 166 / 86$ & $104 / 184 / 66$ & 0.4801 & 0.4463 & 0.0614 & Recessive & 0.9829 \\
\hline rs13340012 & S6 & Intergenic & A & $C$ & $211 / 117 / 22$ & $201 / 134 / 14$ & 0.2300 & 0.2321 & 0.1738 & Recessive & 1 \\
\hline rs 220271 & S7 & Intron & $C$ & $\mathrm{~T}$ & $128 / 164 / 61$ & $135 / 175 / 45$ & 0.4051 & 0.3732 & 0.0860 & Recessive & 0.9955 \\
\hline rs 220276 & S8 & Intron & G & A & $118 / 168 / 66$ & $129 / 168 / 52$ & 0.4261 & 0.3897 & 0.1650 & Allelic & 1 \\
\hline rs 220278 & S9 & Intron & G & A & $143 / 157 / 53$ & $159 / 153 / 42$ & 0.3725 & 0.3347 & 0.1374 & Allelic & 0.9997 \\
\hline rs220281 & $\mathrm{S} 10$ & Intron & G & A & $157 / 151 / 44$ & $149 / 169 / 38$ & 0.3395 & 0.3441 & 0.4408 & Genotypic & 1 \\
\hline rs 220282 & $\mathrm{~S} 11$ & Intron & G & A & $139 / 160 / 55$ & $126 / 160 / 64$ & 0.3814 & 0.4114 & 0.2487 & Allelic & 1 \\
\hline rs 220285 & $\mathrm{~S} 12$ & Intron & $C$ & G & $252 / 69 / 11$ & 269/78/7 & 0.1370 & 0.1299 & 0.2740 & Recessive & 1 \\
\hline rs220298 & $\mathrm{S} 13$ & Intron & G & A & $156 / 159 / 39$ & $181 / 144 / 31$ & 0.3347 & 0.2893 & 0.0647 & Allelic & 0.9867 \\
\hline rs220299 & S14 & Intron & $\mathrm{T}$ & $C$ & $156 / 152 / 46$ & $141 / 168 / 43$ & 0.3446 & 0.3608 & 0.2804 & Dominant & 1 \\
\hline rs8133951 & $\mathbf{S 1 5}$ & Intron & G & A & $126 / 151 / 51$ & $135 / 159 / 48$ & 0.3857 & 0.3728 & 0.5810 & Recessive & 1 \\
\hline rs749020 & $\mathrm{S} 16$ & Intron & G & A & $112 / 166 / 76$ & $112 / 166 / 75$ & 0.4492 & 0.4476 & 0.9425 & Recessive & 1 \\
\hline rs220301 & S17 & Intron & G & $C$ & $136 / 156 / 60$ & $134 / 166 / 50$ & 0.3920 & 0.3800 & 0.3145 & Recessive & 1 \\
\hline rs2839466 & $\mathrm{S} 18$ & Intron & G & A & $136 / 165 / 48$ & $147 / 160 / 43$ & 0.3739 & 0.3514 & 0.3803 & Additive & 1 \\
\hline rs220308 & $\mathbf{S 1 9}$ & Intron & $\mathrm{T}$ & G & $105 / 169 / 80$ & $111 / 168 / 74$ & 0.4647 & 0.4476 & 0.5187 & Allelic & 1 \\
\hline rs220109 & S20 & Exon (syn) & $C$ & $\mathrm{~T}$ & $112 / 153 / 83$ & $114 / 167 / 72$ & 0.4583 & 0.4405 & 0.2706 & Recessive & 1 \\
\hline rs 220110 & S21 & Intron & $C$ & A & $159 / 141 / 52$ & $149 / 154 / 46$ & 0.3480 & 0.3524 & 0.5088 & Dominant & 1 \\
\hline rs12626854 & S22 & Intron & $\mathrm{T}$ & $C$ & $156 / 156 / 41$ & $149 / 156 / 40$ & 0.3371 & 0.3420 & 0.7891 & Dominant & 1 \\
\hline rs 220120 & S23 & Intron & G & $C$ & $165 / 150 / 37$ & $180 / 136 / 34$ & 0.3182 & 0.2914 & 0.2276 & Dominant & 1 \\
\hline rs220131 & S24 & Intron & $C$ & $\mathrm{~T}$ & 86/177/79 & $97 / 171 / 83$ & 0.4898 & 0.4801 & 0.4574 & Dominant & 1 \\
\hline rs220136 & S25 & Intron & $C$ & A & $90 / 185 / 76$ & $92 / 169 / 87$ & 0.4801 & 0.4928 & 0.2953 & Recessive & 1 \\
\hline rs11701944 & S26 & Intron & A & G & $144 / 152 / 48$ & $133 / 169 / 46$ & 0.3605 & 0.3750 & 0.3282 & Dominant & 1 \\
\hline rs 220140 & S27 & Intron & $C$ & G & $161 / 156 / 38$ & $164 / 161 / 31$ & 0.3268 & 0.3132 & 0.3686 & Recessive & 1 \\
\hline rs220143 & S28 & Intron & G & A & $177 / 140 / 35$ & $183 / 143 / 23$ & 0.2983 & 0.2708 & 0.1071 & Recessive & 0.9990 \\
\hline rs2839468 & S29 & Intron & A & $C$ & $111 / 161 / 82$ & 100/179/72 & 0.4590 & 0.4601 & 0.3391 & Genotypic & 1 \\
\hline rs220145 & $\mathrm{S} 30$ & Intron & G & $A$ & $224 / 105 / 18$ & 204/135/17 & 0.2032 & 0.2374 & 0.0489 & Dominant & 0.9569 \\
\hline rs13047454 & S31 & Intron & A & G & $183 / 138 / 34$ & $175 / 160 / 19$ & 0.2901 & 0.2797 & 0.0331 & Recessive & 0.8971 \\
\hline rs220148 & S32 & Intron & A & $C$ & $167 / 146 / 39$ & $182 / 140 / 28$ & 0.3182 & 0.2800 & 0.1182 & Allelic & 0.9995 \\
\hline rs220149 & S33 & Intron & $\mathrm{T}$ & G & $96 / 165 / 86$ & $96 / 185 / 71$ & 0.4856 & 0.4645 & 0.1439 & Recessive & 0.9999 \\
\hline rs 220153 & S34 & Intron & G & A & $212 / 108 / 27$ & $199 / 125 / 23$ & 0.2334 & 0.2464 & 0.3153 & Dominant & 1 \\
\hline rs220154 & S35 & Intron & $C$ & $\mathrm{~T}$ & 109/163/77 & 109/178/64 & 0.4542 & 0.4359 & 0.2066 & Recessive & 1 \\
\hline rs220155 & S36 & Intron & $C$ & $\mathrm{~T}$ & $136 / 144 / 58$ & $125 / 162 / 55$ & 0.3846 & 0.3977 & 0.3229 & Dominant & 1 \\
\hline rs11910495 & S37 & Intron & A & G & $256 / 90 / 8$ & $250 / 92 / 9$ & 0.1497 & 0.1567 & 0.7161 & Allelic & 1 \\
\hline rs13051533 & S38 & Intron & G & A & $169 / 136 / 40$ & $163 / 147 / 34$ & 0.3130 & 0.3125 & 0.4684 & Recessive & 1 \\
\hline rs220157 & S39 & Intron & $C$ & $\mathrm{~T}$ & $126 / 156 / 64$ & $121 / 175 / 47$ & 0.4104 & 0.3921 & 0.0870 & Recessive & 0.9957 \\
\hline rs9984766 & S40 & Intron & G & A & $226 / 86 / 21$ & 204/98/16 & 0.1922 & 0.2044 & 0.3168 & Dominant & 1 \\
\hline rs220158 & S41 & Exon (syn) & $C$ & $\mathrm{~T}$ & $214 / 117 / 23$ & $225 / 104 / 24$ & 0.2302 & 0.2153 & 0.3677 & Dominant & 1 \\
\hline rs220159 & S42 & Exon (ns) & G & A & $136 / 153 / 64$ & $120 / 177 / 54$ & 0.3980 & 0.4060 & 0.1664 & Genotypic & 1 \\
\hline rs2839470 & S43 & Intron & $C$ & $\mathrm{~T}$ & $123 / 163 / 57$ & $125 / 168 / 55$ & 0.4038 & 0.3994 & 0.7717 & Recessive & 1 \\
\hline
\end{tabular}


Table 2 Summary statistics of UMODL1 SNPs in initial study (cases defined as spherical equivalent $\leq-8.0$ diopters) (Continued)

\begin{tabular}{|c|c|c|c|c|c|c|c|c|c|c|c|}
\hline rs4920063 & S44 & Intron & G & A & $155 / 139 / 47$ & 133/184/36 & 0.3416 & 0.3626 & 0.0100 & Genotypic & 0.9219 \\
\hline rs220161 & S45 & Intron & C & G & $92 / 158 / 83$ & $106 / 171 / 67$ & 0.4865 & 0.4433 & 0.0879 & Recessive & 0.9963 \\
\hline rs2839471 & S46 & Intron & $\mathrm{T}$ & C & $98 / 178 / 76$ & $97 / 187 / 70$ & 0.4688 & 0.4619 & 0.5512 & Recessive & 1 \\
\hline rs220168 & S47 & Intron & $A$ & G & $217 / 113 / 21$ & $198 / 132 / 21$ & 0.2208 & 0.2479 & 0.1447 & Dominant & 0.9999 \\
\hline rs220170 & S48 & Intron & C & $\mathrm{T}$ & 212/123/19 & $199 / 130 / 23$ & 0.2274 & 0.2500 & 0.3192 & Allelic & 1 \\
\hline rs11911271 & S49 & Intron & $\mathrm{T}$ & C & $176 / 148 / 27$ & $201 / 122 / 31$ & 0.2877 & 0.2599 & 0.0773 & Dominant & 0.9929 \\
\hline rs220171 & $\mathrm{S} 50$ & Intron & C & $\mathrm{T}$ & $158 / 163 / 30$ & $185 / 137 / 30$ & 0.3177 & 0.2798 & 0.0455 & Dominant & 0.9477 \\
\hline rs220172 & S51 & Intron & G & A & $171 / 137 / 34$ & $160 / 139 / 35$ & 0.2997 & 0.3129 & 0.5858 & Dominant & 1 \\
\hline rs220173 & S52 & Intron & C & G & $88 / 183 / 84$ & $97 / 180 / 79$ & 0.4944 & 0.4747 & 0.4535 & Additive & 1 \\
\hline rs3819141 & S53 & Exon (syn) & $\mathrm{T}$ & A & $128 / 163 / 62$ & $120 / 160 / 73$ & 0.4065 & 0.4334 & 0.2925 & Recessive & 1 \\
\hline rs3819142 & S54 & Exon (ns) & $A$ & C & 206/130/19 & 206/130/19 & 0.2366 & 0.2514 & 0.5125 & Additive & 1 \\
\hline rs915840 & S56 & Intron & $\mathrm{T}$ & C & $212 / 122 / 21$ & $212 / 122 / 21$ & 0.2310 & 0.2486 & 0.3025 & Dominant & 1 \\
\hline rs220181 & S57 & Intron & G & A & $183 / 140 / 15$ & 183/140/15 & 0.2515 & 0.2536 & 0.2039 & Recessive & 1 \\
\hline
\end{tabular}

*SNPs are listed down the column in sequential order from the $5^{\prime}$ end to the $3^{\prime}$ end of the sense strand of the UMODL 1 gene. They are also designated as S1 to S57 for the sake of easy referencing. SNPs located in exons are either synonymous (syn) or non-synonymous (ns).

†1: major allele; 2 : minor allele.

Single-marker analysis is performed with Plink. Asymptotic $P$ values $\left(P_{\text {asym }}\right)$ are obtained by chi-square test, and empirical $P$ values $\left(P_{\text {emp }}\right)$ obtained by 10,000 permutations for correcting multiple comparisons. The genetic models tested for each SNP are allelic, genotypic, additive (tested by trend test), dominant and recessive. Here, only the best result and the corresponding genetic model are shown for each SNP.

rs3819142 (S54) gave the best $P$ value (0.0055) with OR= 0.68 (Table 3) among other haplotypes. Single-marker analysis by Beagle produced a marginally significant result $\left(P_{\mathrm{emp}}=0.0569\right)$ for the $\mathrm{C}$ allele of rs3819142 (S54). This implied that the association signal was not robust. Moreover, combined analysis of both sample sets for cases with $\mathrm{SE} \leq-10 \mathrm{D}$ did not reveal this association signal. Consistent with the analysis by Plink, overall analysis by Beagle indicated that rs3819142 (S54) was not associated with high myopia. In summary, the putatively positive results of each sample set could not been replicated by the other sample set as well as the combined sample set.

\section{Association analysis of secondary phenotypes}

In order to fully explore the ocular data and genotype data we had obtained, we conducted regression association analysis of secondary quantitative phenotypes (AXL, ACD, LT and CP) for Sample Set 1. No significant association was observed between these quantitative traits and any of the SNPs tested, except rs220120 (S23). Association of rs220120 (S23) with anterior chamber depth (ACD) was demonstrated for samples with cases defined by $\mathrm{SE} \leq-8$ D: $P_{\text {asym }}=0.001$ and FDR-adjusted $P=0.046$. The regression coefficient for the risk allele $\mathrm{C}$ of rs220120 (S23) was -0.066 (95\% CI: -0.104 to -0.027 ). This means that each copy increase of the risk allele $\mathrm{C}$ decreased the anterior chamber depth by $0.066 \mathrm{~mm}$ on average. The association signal was stronger for a subset of Sample Set 1 with cases defined by SE $\leq-10 \mathrm{D}: P_{\text {asym }}=0.0003$ and FDR-adjusted
$P=0.019$ with the corresponding regression coefficient being -0.080 (95\% CI: -0.124 to -0.036$)$.

\section{Discussion}

The $U M O D L 1$ gene was first revealed as a new susceptibility gene for high myopia in a recent Japanese casecontrol study [8]. In particular, the SNP rs2839471 (S46) was found to be associated with high myopia (corrected $P=0.01$ and $\mathrm{OR}=1.68$ ). Our study aimed at systematically investigating the $U M O D L 1$ gene for association with high myopia in a Han Chinese population, and served to confirm or refute the original Japanese study. We genotyped and analyzed Sample Set 1 (Table 1) for 57 SNPs selected from the $86-\mathrm{kb}$ region encompassing the UMODL1 locus. Using five genetic models and cases defined by three different SE thresholds, single-marker analysis did not reveal any significant association between any single SNPs and high myopia after controlling for multiple comparisons. The conclusion remained the same no matter whether sex and age were included as covariates or not.

Haplotype-based analysis is more powerful in detecting association than single-marker analysis [27]. Different approaches of haplotype analysis offer different merits and disadvantages [22,28]. We performed haplotype analysis of our genetic data with three methods: LD-block-based method by Haploview, exhaustive variable-sized sliding window strategy by Plink, and localized haplotype clustering approach by Beagle. Of all these approaches, only 
Table 3 Identification of haplotypes in localized haplotype clusters that are associated with high myopia

\begin{tabular}{|c|c|c|c|c|c|c|c|c|}
\hline \multirow[t]{2}{*}{ Markers \& haplotypes* } & \multicolumn{2}{|c|}{ Haplotype counts } & \multicolumn{2}{|c|}{ Haplotype being considered } & \multicolumn{2}{|c|}{ All other haplotypes } & \multicolumn{2}{|c|}{ Fisher's exact test } \\
\hline & Total no.t & no. in cluster (\%) & Controlst & Casest & Controls & Cases & $P$ value $\ddagger$ & OR $(95 \% \mathrm{Cl}) \S$ \\
\hline \multicolumn{9}{|c|}{ Sample Set 1 for cases defined by SE $\leq-12$ diopters Marker $=$ rs220168 (S47), Cluster $=0 . G$} \\
\hline \multicolumn{9}{|l|}{ Markers: S47 } \\
\hline G (2) & 202 & $28(13.9 \%)$ & 155 & 47 & 557 & 135 & 0.2744 & $1.25(0.83-1.85)$ \\
\hline \multicolumn{9}{|l|}{ Markers: S47-\$48 } \\
\hline GC (21) & 35 & $26(74.3 \%)$ & 22 & 13 & 690 & 169 & 0.0176 & $2.41(1.09-5.12)$ \\
\hline GT (22) & 167 & $2(1.2 \%)$ & 133 & 34 & 579 & 148 & 1.0000 & $1.00(0.63-1.54)$ \\
\hline \multicolumn{9}{|l|}{ Markers: S47-S48-\$49 } \\
\hline GCT (211) & 25 & $25(100.0 \%)$ & 13 & 12 & 699 & 170 & 0.0015 & $3.78(1.54-9.19)$ \\
\hline GCC (212) & 10 & $1(10.0 \%)$ & 9 & 1 & 703 & 181 & 0.6965 & $0.43(0.01-3.15)$ \\
\hline GTC (222) & 23 & $2(8.7 \%)$ & 17 & 6 & 695 & 176 & 0.4411 & $0.39(0.44-3.77)$ \\
\hline \multicolumn{9}{|l|}{ Markers: S47-S48-S49-S50 } \\
\hline GCTC (2112) & 13 & $13(100.0 \%)$ & 8 & 5 & 704 & 177 & 0.1546 & $2.48(0.63-8.73)$ \\
\hline GCTT (2111) & 12 & $12(100.0 \%)$ & 5 & 7 & 707 & 175 & 0.0041 & $5.64(1.52-22.82)$ \\
\hline GCCC (2122) & 10 & $1(10.0 \%)$ & 9 & 1 & 703 & 181 & 0.6965 & $0.43(0.01-3.15)$ \\
\hline GTCC (2222) & 3 & $2(66.7 \%)$ & 1 & 2 & 711 & 180 & 0.1071 & $7.87(0.41-465.3)$ \\
\hline
\end{tabular}

Sample Set 2 for cases defined by SE $\leq-10$ diopters Marker $=$ rs3819142 (S54), Cluster $=0 . C$

Markers: S54

C (2) 290

$222(76.6 \%)$

193

97

595

441

0.0055

$0.68(0.51-0.90)$

Markers: S49-S54

TC (12)

253

$214(84.6 \%)$

166

87

622

451

0.0274

$0.72(0.54-0.97)$

CC (22)

$37 \quad 8(21.6 \%)$

$10 \quad 761$

$528 \quad 0.0926$

$0.43(0.23-1.15)$

*Markers are rs220168 (S47), rs220170 (S48), rs11911271 (S49), rs220171 (S50) and rs3819142 (S54) along the 5' end to the 3' end direction of the sense strand of the UMODL1 gene. A localized haplotype cluster is indicated in the format of "(node number).(allele of the marker being considered)". For example,

"Marker =rs220168 (S47), Cluster = 0.G" refers to the G allele of the marker S47 at node "0" (zero). The allele of each marker is shown in both the ACGT and the 1-2 formats ( 1 being the major and 2 the minor allele). For each localized haplotype cluster found to be associated with high myopia by Beagle, the table here shows the haplotypes for an increasing number of markers until the haplotypes account for all or almost all of the association signals for the cluster and give the lowest $P$ values (in boldface) for the Fisher's exact test. Then, haplotypes are shown for one extra window with one additional marker just to show that one additional marker does not provide additional information and the $P$ values for Fisher's exact test become less impressive (i.e. larger).

†For a particular haplotype under consideration, the total count is the sum of the counts in both controls and cases.

Note that the $P$ values for the Fisher's exact test are not corrected for multiple testing because the cluster2haps program is only used to identify the haplotypes involved in the haplotype clusters that are already found positive by Beagle after adjustment by permutations for multiple comparisons.

\$The odds ratio (OR) is calculated for the haplotype being considered with regard to all other haplotypes as the reference. The $95 \%$ confidence intervals (CI) are also shown.

Beagle identified a marginally significant $\left(P_{\mathrm{emp}}=0.0763\right)$ haplotype cluster (0.G) for rs220168 (S47) for Sample Set 1 with cases defined by $\mathrm{SE} \leq-12 \mathrm{D}$. Further analysis showed that three SNPs contributed to this haplotype association signal: rs220168 (S47), rs220170 (S48) and rs11911271 (S49) (Table 3). However, we failed to replicate this association signal with a second bigger sample set (Sample Set 2; Table 2) with all attempts (different SE thresholds for defining cases, and with or without adjustment for potential confounding by sex and age).

Instead, a non-synonymous SNP (rs3819142 or S54) showed significant association with high myopia for Sample Set 2 with cases defined by SE $\leq-10$ D (Tables 3 and 4). This non-synonymous SNP was added in the follow-up study because it was in moderate LD $\left(r^{2}=0.6\right)$ with rs220170 (S48), which was one of the SNPs contributing to the positive haplotype cluster revealed in
Sample Set 1. However, we failed to confirm the association signal of rs3819142 (S54) back in Sample Set 1 and in the combined sample set (Sets 1 and 2 combined), even with different strategies of haplotype analysis.

Overall, our results indicated that common polymorphisms in the UMODL1 gene were not associated with susceptibility to high myopia in the Chinese population in Hong Kong. Power analysis strengthens the credibility of a genetic association study [5]. Therefore, power analysis is carried out using QUANTO (ver. 1.2.3) [29] for our study. We assume that the prevalence of high myopia is 0.05 in the Chinese population in Hong Kong [30], OR $=1.68$ as obtained in the original Japanese study for the positive SNP rs2839471 (S46) and MAF = 0.2 (the cutoff for SNP selection for this study). We set the significance level at $0.00088(=0.05 / 57)$ because 57 SNPs were analyzed for possible association with high 
Table 4 Summary statistics of UMODL1 SNPs in follow-up study (cases defined as spherical equivalent $\leq-10.0$ diopters)

\begin{tabular}{|c|c|c|c|c|c|c|c|c|c|c|c|}
\hline \multirow{2}{*}{ SNP* } & & \multirow[b]{2}{*}{ Location* } & \multicolumn{2}{|c|}{ Allelet } & \multicolumn{2}{|c|}{ Genotype counts $(11 / 12 / 22)$} & \multicolumn{2}{|c|}{ Minor allele freq } & \multicolumn{3}{|c|}{ Association test (best result) $\ddagger$} \\
\hline & & & 1 & 2 & Controls & Cases & Controls & Cases & $P_{\text {asym }}$ & Model & $P_{\text {emp }}$ \\
\hline \multicolumn{12}{|c|}{ Follow-up study (Sample Set 2) } \\
\hline rs220168 & S47 & Intron & A & G & $220 / 138 / 22$ & $179 / 712 / 12$ & 0.2395 & 0.1813 & 0.0074 & Dominant & 0.0568 \\
\hline rs220170 & S48 & Intron & C & $\mathrm{T}$ & $228 / 120 / 22$ & $178 / 70 / 15$ & 0.2216 & 0.1901 & 0.1173 & Dominant & 0.5556 \\
\hline rs11911271 & S49 & Intron & $\mathrm{T}$ & C & $205 / 157 / 27$ & $138 / 117 / 14$ & 0.2712 & 0.2695 & 0.7241 & Dominant & 0.9536 \\
\hline rs3819142 & S54 & Exon (ns) & A & $\mathrm{C}$ & $215 / 149 / 20$ & $182 / 76 / 10$ & 0.2461 & 0.1791 & 0.0021 & Dominant & 0.0168 \\
\hline \multicolumn{12}{|c|}{ Combined analysis (Sample Sets 1 and 2) } \\
\hline rs220168 & S47 & Intron & A & G & $436 / 251 / 43$ & $291 / 149 / 24$ & 0.2308 & 0.2123 & 0.2883 & Allelic & 0.8884 \\
\hline rs220170 & S48 & Intron & C & $\mathrm{T}$ & $439 / 243 / 41$ & $294 / 145 / 27$ & 0.2248 & 0.2135 & 0.4118 & Dominant & 0.9691 \\
\hline rs11911271 & S49 & Intron & $\mathrm{T}$ & C & $380 / 305 / 54$ & $256 / 183 / 32$ & 0.2794 & 0.2622 & 0.3194 & Dominant & 0.9242 \\
\hline rs3819142 & S54 & Exon (ns) & A & C & $420 / 279 / 39$ & $294 / 154 / 23$ & 0.2419 & 0.2123 & 0.0574 & Dominant & 0.3303 \\
\hline
\end{tabular}

*SNPs are listed down the column in sequential order from the $5^{\prime}$ end to the $3^{\prime}$ end of the sense strand of the UMODL 1 gene. They are also designated as S47 to S49 and S52 as explained in the footnote to Table 2. The non-synonymous (ns) SNP rs3819142 (S54) is located in exon.

†1: major allele; 2 : minor allele.

Single-marker analysis is performed with Plink. Asymptotic $P$ values $\left(P_{\text {asym }}\right)$ are obtained by chi-square test, and empirical $P$ values $\left(P_{\text {emp }}\right)$ obtained by 10,000 permutations for correcting multiple comparisons. The genetic models tested for each SNP are allelic, genotypic, additive (tested by trend test), dominant and recessive. Here, only the best result and the corresponding genetic model are shown for each SNP.

myopia in the initial study. With a sample size of 356 controls and 356 cases, our initial study has $\sim 80 \%$ power of detecting association under a log-additive genetic model. The power is almost $95 \%$ when the MAF is $\sim 0.45$ - the MAF of rs2839471 (S46) in the present study. Note that the power is expected to be less under other genetic models. This power analysis is valid only for single-marker analyses and a single phenotype (say, high myopia defined by a threshold of $-8 \mathrm{D}$ ). We are also aware that the association analysis has been performed (1) for many more markers as defined by haplotypes and (2) many more phenotypes based on different thresholds for defining high myopia, and ocular quantitative traits (secondary phenotypes). The abovementioned power analysis does not take into account these multiple markers and phenotypes. Although the permutation procedures of the analysis packages can handle both single markers and haplotypes together, they do not take into account the issues of multiple phenotypes. This will thus increase the false positive rates of the initial study. However, the main purpose of the initial study was to identify potential significant results so that they could be further examined in the follow-up study. The follow-up study used a larger sample size of 394 controls and 526 cases and examined 4 SNPs with 3 different definitions of high myopia and three different software packages. Assuming naively that the number of comparisons increases linearly with the number of multiple phenotypes examined by different packages, Quanto predicts that the power of the follow-up study is still well above $80 \%$.

In our initial study, the SNP (rs2839471 or S46) originally positive in the Japanese study [8] was negative in all the analyses. The original Japanese study genotyped

\begin{tabular}{|c|c|c|c|c|c|c|}
\hline $\begin{array}{l}\text { Study } \\
\text { or sub-category }\end{array}$ & $\begin{array}{l}\text { Case } \\
\mathrm{nN}\end{array}$ & $\begin{array}{c}\text { Control } \\
\mathrm{nN}\end{array}$ & $\begin{array}{c}\text { OR (random) } \\
95 \% \mathrm{Cl}\end{array}$ & $\begin{array}{c}\text { Weight } \\
\%\end{array}$ & & $\begin{array}{l}\text { OR (random) } \\
\quad 95 \% \mathrm{Cl}\end{array}$ \\
\hline Japanese study & $530 / 1040$ & $461 / 1034$ & $\Rightarrow$ & 32.26 & 1.29 & {$[1.09,1.54]$} \\
\hline Zhongshan's study & $1877 / 3636$ & $1102 / 2104$ & 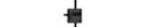 & 39.37 & 0.97 & {$[0.87,1.08]$} \\
\hline Present study & $381 / 708$ & $374 / 704$ & $-\frac{1}{2}$ & 28.36 & 1.03 & {$[0.83,1.27]$} \\
\hline $\begin{array}{l}\text { Total }(95 \% \mathrm{Cl}) \\
\text { Total events: } 2788(\mathrm{C} \\
\text { Test for heterogeneit } \\
\text { Test for overall effec }\end{array}$ & $\begin{array}{l}5384 \\
=2(P=0.02) \\
\text { itrol) } \\
39)\end{array}$ & 3842 & & 100.00 & 1.08 & {$[0.90,1.30]$} \\
\hline
\end{tabular}

Figure 1 Meta-analysis of case-control studies examining the association between high myopia and rs2839471 within UMODL1. Three studies are combined: the original Japanese study [8], a very recent Chinese study (Zhongshan's study) [27], and the present study. The number (n) of the T allele of rs 2839471 and the total number ( $N$ ) of alleles (T and C) in the case group and the control group are shown in the second and third columns from the left. Note that rs2839471 (S46) was genotyped only in Sample Set 1 in the present study; there were 354 cases and 352 controls (see Table 2), and hence a total of 708 alleles in cases and 704 alleles in controls because 6 samples failed to be genotyped by iPLEX assays. The allelic odds ratios (OR) are shown diagrammatically in the middle, and numerically in the last column on the right. The contribution from each study is proportional to the size of the black square in the middle, and the exact weight is indicated in the second last column from the right. Overall, meta-analysis does not support the association between high myopia and rs2839471 ( $P=0.39$, DerSimonian and Laird method). 
39 SNPs for 520 controls and 520 cases with $\mathrm{SE}<-9.25$ D [8], and 23 of these SNPs were within the same region examined by us. Of these 23 SNPs, 17 were also genotyped in our initial study. The remaining 6 SNPs were not tested in the present study because they did not satisfy the criteria of selecting tag SNPs - with an MAF $<0.20$ or not documented in the Han Chinese data of the HapMap Project. A very recent study only examined a single SNP (rs2839471 or S46) of the UMODL1 gene for a very large group of unrelated Chinese subjects $(\mathrm{n}=2,870)$ and reported no association between this SNP and moderate/high myopia (defined by different thresholds of spherical refraction at $-4 \mathrm{D}$ and $-6 \mathrm{D}$ ) [31]. Therefore, we carry out meta-analysis to combine the data from all three studies for the SNP rs2839471 (S46) - the putatively positive SNP first identified by the Japanese study. For this purpose, we use the RevMan program (ver. 5.1.6) [32]. We note that different thresholds of SE or spherical refraction were used to define controls and cases with high myopia in these three studies. Therefore, we use a random-effects model for combining the data, as is supported by a significant test for heterogeneity $(P=0.02$, Figure 1$)$. Meta-analysis supports that there is no association between rs2839471 and high myopia: $P=0.39$ (DerSimonian and Laird method for overall effect), and allelic $\mathrm{OR}=1.08$ (95\% CI, $0.90-$ 1.30) for allele $T$ with reference to allele $C$ (Figure 1).

Analysis of secondary phenotypes collected during case-control studies can provide valuable insights into biological pathways and help in identifying the association between genetic variants and phenotypes [25]. In the present study, no significant differences in AXL, CP and LT were found among different genotype groups in the case-control subjects. Instead, rs220120 (S23) showed association with ACD $\left(P_{\text {asym }}=0.001, \quad\right.$ FDR-adjusted $P=0.046)$. Intriguingly, this SNP is predicted to affect the binding of transcription factors (http://manticore. niehs.nih.gov/snpfunc.htm) and hence may affect the expression of UMODL1. However, the association signal may also be driven by a causal variant in strong LD with this SNP. If the association signal turns out to be genuine upon replication, it is worth examining the biological roles of the causal variant and UMODL1 in influencing ACD.

Refraction is determined by AXL, CP, LT and ACD. Of these, AXL is the primary determinant of high myopia, but not ACD [33]. Both AXL and ACD are highly heritable traits (heritability 0.67 for AXL and 0.78 for ACD) and are strongly correlated to each other [34]. While some of the genetic factors may be shared between AXL and $\mathrm{ACD}$, others are unique to ACD $[34,35]$. Therefore, it is possible that the significant SNP for ACD is not associated with refraction. A genome-wide linkage study had identified $1 \mathrm{p} 32$ as a susceptibility locus for ACD [36]. Our results indicated that UMODL1 might be another candidate gene for ACD, but not AXL. Note that we did not take multiple secondary phenotypes into account while adjusting for multiple testing of SNPs. Therefore, the results of this exploratory study must be regarded as preliminary findings and need to be confirmed by additional studies.

\section{Conclusions}

In conclusion, we systematically investigated the common polymorphisms of the UMODL1 gene for association with high myopia by genotyping 59 SNPs in the initial study and 4 SNPs in the follow-up study. Both single-marker and haplotype analyses did not demonstrate any association with high myopia. However, exploratory association analysis of secondary phenotypes indicated the rs220120 was associated with ACD. We suggested that common polymorphisms of the UMODL1 gene were unlikely to play an important role in the genetic susceptibility to high myopia in the Chinese population under study, and the role of UMODL1 in ACD remained to be confirmed.

\section{Additional files}

Additional file 1: Primers for genotyping SNPs by MassARRAY iPLEX (Sequenom). This table summarizes the primer sequences for genotyping SNPs by MassARRAY iPLEX (Sequenom). SNPS are arranged down the column in the sequential order from the $5^{\prime}$ end to the $3^{\prime}$ end of the UMODL1 gene.

Additional file 2: PCR Primers and conditions for genotyping SNPs by RFLP and DHPLC. This table summarizes the PCR primers and conditions for genotyping SNPs by RFLP and DHPLC. The restriction enzyme and the concentration of polyacrylamide gel used are indicated for each SNP being genotyped by RFLP. The primer for primer extension is shown for SNP genotyped by DHPLC. Some primers have a poly-T or poly-A tail at the $5^{\prime}$ end to enhance the difference in the size of restricted fragments for easy genotype calling.

Additional file 3: The gene structure and linkage disequilibrium (LD) pattern of the UMODL1 gene. (A) The top panel shows the physical positions on chromosome 21, and the exon-intron organization for the isoforms of the UMODL1 gene at 21q22.3. (B) The bottom panel shows the distribution of 57 SNPs analyzed in this study and LD blocks as defined by the solid spine of LD algorithm of Haploview. The LD measures are indicated as $r^{2}$ values. The intensities of the red colour indicate the magnitude with deep red being a value of $100 \%$ or 1 for the $r^{2}$ value.

\section{Abbreviations}

SNPs, Single nucleotide polymorphisms; UMODL1, The uromodulin-like 1 gene; $A X L$, Axial length; SE, Spherical equivalent; ACD, Anterior chamber depth; LT, Lens thickness; CP, Corneal power; MAF, Minor allele frequency; $L D$, Linkage disequilibrium; OR, Odds ratios; RFLP, Restriction fragment length polymorphism; DHPLC, Denaturing high performance liquid chromatography; PCR, Polymerase chain reaction; HWE, Hardy-Weinberg equilibrium; FDR, False discovery rate; SSLD, Solid spine of linkage disequilibrium; $P_{\text {emp }}$, Empirical $P$ values; $P$ asym, Asymptotic $P$ value.

\section{Competing interests}

The authors declare that they have no competing interests. 


\section{Authors' contributions}

MMZ carried out genotyping work, performed statistical analysis of single-marker and haplotype association analysis, and drafted the manuscript. MKH and YSG participated in the design of the study. DWHH carried out statistical analysis of secondary phenotypes. PWN recruited study subjects, and performed all the ocular examination. WYF processed blood samples and extracted DNA. SPY conceived, designed and coordinated the whole study, drafted and finalized the manuscript. All authors read and approved the final manuscript.

\section{Acknowledgments}

The work was supported by grants from The Hong Kong Polytechnic University (G-U788, J-BB7P, 87TP and 87LV). The study is a contribution from the Centre for Myopia Research, The Hong Kong Polytechnic University. The authors would like to thank all subjects participating in the Myopia Genetics Study.

\section{Author details}

${ }^{1}$ Department of Ophthalmology, The First Affiliated Hospital, Zhejiang University School of Medicine, Hangzhou, China. ${ }^{2}$ Department of Health Technology and Informatics, The Hong Kong Polytechnic University, Hong Kong SAR, China. ${ }^{3}$ Centre for Myopia Research, School of Optometry, The Hong Kong Polytechnic University, Hong Kong SAR, China.

Received: 9 February 2012 Accepted: 27 July 2012

Published: 2 August 2012

\section{References}

1. Saw SM, Gazzard G, Shih-Yen EC, Chua WH: Myopia and associated pathological complications. Ophthalmic Physiol Opt 2005, 25(5):381-391.

2. Vitale S, Sperduto RD, Ferris FL 3rd: Increased prevalence of myopia in the United States between 1971-1972 and 1999-2004. Arch Ophthalmol 2009, 127(12):1632-1639.

3. Kempen JH, Mitchell P, Lee KE, Tielsch JM, Broman AT, Taylor HR, Ikram MK Congdon NG, O'Colmain BJ: The prevalence of refractive errors among adults in the United States, Western Europe, and Australia. Arch Ophthalmol 2004, 122(4):495-505

4. Saw SM, Chua WH, Wu HM, Yap E, Chia KS, Stone RA: Myopia: geneenvironment interaction. Ann Acad Med Singapore 2000, 29(3):290-297.

5. Tang WC, Yap MK, Yip SP: A review of current approaches to identifying human genes involved in myopia. Clin Exp Optom 2008, 91(1):4-22.

6. Dirani M, Chamberlain M, Garoufalis P, Chen C, Guymer RH, Baird PN: Refractive errors in twin studies. Twin Res Hum Genet 2006, 9(4):566-572.

7. Lopes MC, Andrew T, Carbonaro F, Spector TD, Hammond CJ: Estimating heritability and shared environmental effects for refractive error in twin and family studies. Invest Ophthalmol Vis Sci 2009, 50(1):126-131.

8. Nishizaki R, Ota M, Inoko H, Meguro A, Shiota T, Okada E, Mok J, Oka A, Ohno S, Mizuki N: New susceptibility locus for high myopia is linked to the uromodulin-like 1 (UMODL1) gene region on chromosome 21q22.3. Eye (Lond) 2009, 23(1):222-229.

9. Shibuya K, Nagamine K, Okui M, Ohsawa Y, Asakawa S, Minoshima S, Hase T, Kudoh J, Shimizu N: Initial characterization of an uromodulin-like 1 gene on human chromosome 21q22.3. Biochem Biophys Res Commun 2004 , 319(4):1181-1189.

10. Di Schiavi E, Riano E, Heye B, Bazzicalupo P, Rugarli El: UMODL1/Olfactorin is an extracellular membrane-bound molecule with a restricted spatial expression in olfactory and vomeronasal neurons. Eur J Neurosci 2005, 21(12):3291-3300.

11. EyeBrowse; http://eyebrowse.cit.nih.gov/.

12. Zha Y, Leung KH, Lo KK, Fung WY, Ng PW, Shi MG, Yap MK, Yip SP: TGFB1 as a susceptibility gene for high myopia: a replication study with new findings. Arch Ophthalmol 2009, 127(4):541-548.

13. Barrett JC, Fry B, Maller J, Daly MJ: Haploview: analysis and visualization of LD and haplotype maps. Bioinformatics 2005, 21(2):263-265.

14. International HapMap Project; http://www.hapmap.org/

15. Oeth P, del Mistro G, Marnellos G, Shi T, van den Boom D: Qualitative and quantitative genotyping using single base primer extension coupled with matrix-assisted laser desorption/ionization time-of-flight mass spectrometry (MassARRAY). Methods Mol Biol 2009, 578:307-343.
16. Leung KH, Yip SP: Denaturing high-performance liquid chromatography In Molecular Biomethods Handbook. 2nd edition. Edited by Walker JM, Rapley R. Totowa, NJ: Humana Press; 2008:89-106.

17. Leung KH, Yiu WC, Yap MK, Ng PW, Fung WY, Sham PC, Yip SP: Systematic investigation of the relationship between high myopia and polymorphisms of the MMP2, TIMP2 and TIMP3 genes by a DNA pooling approach. Invest Ophthalmol Vis Sci 2011, 52(6):3893-3900.

18. Yip SP, Leung KH, Ng PW, Fung WY, Sham PC, Yap MK: Evaluation of proteoglycan gene polymorphisms as risk factors in the genetic susceptibility to high myopia. Invest Ophthalmol Vis Sci 2011, 52(9):6396-403.

19. Ho DW, Yiu WC, Yap MK, Fung WY, Ng PW, Yip SP: Genotyping performance assessment of whole genome amplified DNA with respect to multiplexing level of assay and its period of storage. PLOS One 2011, 6(10):e26119.

20. Han W, Yip SP, Wang J, Yap MK: Using denaturing HPLC for SNP discovery and genotyping, and establishing the linkage disequilibrium pattern for the all-trans-retinol dehydrogenase (RDH8) gene. J Hum Genet 2004 49(1):16-23.

21. Purcell S, Neale B, Todd-Brown K, Thomas L, Ferreira MA, Bender D, Maller J, Sklar P, de Bakker PI, Daly MJ, Sham PC: PLINK: a tool set for wholegenome association and population-based linkage analyses. Am J Hum Genet 2007, 81(3):559-575.

22. Browning BL, Browning SR: Efficient multilocus association testing for whole genome association studies using localized haplotype clustering. Genet Epidemiol 2007, 31(5):365-375.

23. Browning SR, Browning BL: Rapid and accurate haplotype phasing and missing-data inference for whole-genome association studies by use of localized haplotype clustering. Am J Hum Genet 2007, 81(5):1084-1097.

24. BEAGLE software package. http://faculty.washington.edu/browning/beagle/ beagle.html

25. Lin DY, Zeng D: Proper analysis of secondary phenotype data in casecontrol association studies. Genet Epidemiol 2009, 33(3):256-265.

26. Storey JD, Tibshirani R: Statistical significance for genomewide studies. Proc Natl Acad Sci USA 2003, 100(16):9440-9445.

27. Schaid DJ: Evaluating associations of haplotypes with traits. Genet Epidemiol 2004, 27(4):348-364.

28. Guo Y, Li J, Bonham AJ, Wang Y, Deng H: Gains in power for exhaustive analyses of haplotypes using variable-sized sliding window strategy: a comparison of association-mapping strategies. Eur J Hum Genet 2009, 17(6):785-792.

29. Quanto software package. a. http://hydra.usc.edu/gxe/.

30. Goh WS, Lam CS: Changes in refractive trends and optical components of Hong Kong Chinese aged 19-39 years. Ophthalmic Physiol Opt 1994, 14(4):378-382

31. Wang Q, Gao Y, Wang P, Li S, Jia X, Xiao X, Guo X, Zhang Q: Replication study of significant single nucleotide polymorphisms associated with myopia from two genome-wide association studies. Mol Vis 2011, 17:3290-3299.

32. RevMan software package. http://ims.cochrane.org/revman/download

33. Meng W, Butterworth J, Malecaze F, Calvas P: Axial length of myopia: a review of current research. Ophthalmologica 2011, 225(3):127-134.

34. Klein AP, Suktitipat B, Duggal P, Lee KE, Klein R, Bailey-Wilson JE, Klein BE: Heritability analysis of spherical equivalent, axial length, corneal curvature, and anterior chamber depth in the Beaver Dam Eye Study. Arch Ophthalmol 2009, 127(5):649-655.

35. He M, Hur YM, Zhang J, Ding X, Huang W, Wang D: Shared genetic determinant of axial length, anterior chamber depth, and angle opening distance: the Guangzhou Twin Eye Study. Invest Ophthalmol Vis Sci 2008, 49(11):4790-4794.

36. Biino G, Palmas MA, Corona C, Prodi D, Fanciulli M, Sulis R, Serra A, Fossarello M, Pirastu M: Ocular refraction: heritability and genome-wide search for eye morphometry traits in an isolated Sardinian population. Hum Genet 2005, 116(3):152-159.

doi:10.1186/1471-2350-13-64

Cite this article as: Zhu et al.: Investigating the relationship between UMODL 1 gene polymorphisms and high myopia: a case-control study in Chinese. BMC Medical Genetics 2012 13:64. 